\title{
BIG DATA BUSINESS ANALYTICS AS A STRATEGIC ASSET FOR HEALTH CARE INDUSTRY
}

\author{
Dr. S. Smys, \\ Professor, Department of Computer Science and Engineering, \\ RVS Technical Campus, \\ Coimbatore, India. \\ Email: smys375@gmail.com \\ Mr. C. Vijesh joe, \\ Assistant Professor, Department of Computer Science and Engineering, \\ VV College of Engineering, \\ Tirunelveli, India. \\ Email id: vijesh.joe@gmail.com
}

\begin{abstract}
The big data includes the enormous flow of data from variety of applications that does not fit into the traditional data base. They deal with the storing, managing and manipulating of the data acquired from various sources at an alarming rate to gather valuable insights from it. The big data analytics is used provide with the new and better ideas that pave way to the improvising of the business strategies with its broader, deeper insights and frictionless actions that leads to an accurate and reliable systems. The paper proposes the big data analytics for the improving the strategic assets in the health care industry by providing with the better services for the patients, gaining the satisfaction of the patients and enhancing the customer relationship.
\end{abstract}

Keywords: Big-data analytics, strategic assets, healthcare industry, customer relationship and patient satisfaction.

\section{Introduction}

The big data analytics has become a significant part for the concerns, handling huge volume of data that flow from various departments. Since it helps in the extracting the valuable information's from the huge volume of the data to improvise the performance of the organization. The huge volume and the variety of the data flow analyzed with and extracted for the valuable information's to address the areas that are weak and improvises the agility of the organization that is most important in the exploring of the competitive lead [4]. The big data analytics, in general is portrayed by the complexity of the information's of huge volume, velocity, veracity and variety [6], this big data analytics in beginning emerged. The big data analytics has its part in almost a wide range of industries as the analysis help in ameliorate the strategic assets of the industries. The industries most probably involved in the large scale production, with huge amount of data flow are to employ the big-data analytics, to have a progressive growth in all areas staring from planning, raw material purchase, and designing, developing, producing, marketing and 
Journal of ISMAC (2019)

Vol.01/ No. 02, June 2019, pp: 92-100

DOI: https://doi.org/10.36548/jismac.2019.2.002

ISSN: 2582-1369 (online)

maintaining the customer relationship. So the analytics based on the big data flow ensures the boost the revenue of the company, cutting down the unwanted expense and also presents with the ways of improving the revenue and the customer relationship. The big data is also involved in the health care industry that has a huge amount of data to be processed with to produce productive results in terms of the diagnosis of the disease [7], proper treatment, and better decision making, enhancing the quality of the health care at the reduced cost. The big-data developed holds the potency to cause dramatic change in the procedures of planning and decision- making, improving the mode of business from the huge amount of data obtained and the information gained.

The big-data enables the gathering the of huge amount of information from variety of sources, at a higher alarming rate, with different formats and values contained in it. The big-data analytics or the business analytics allows one to find the newer path to raise his business to heights. The development leading to an improved environment for the business with the enhanced decision making and effective operations to have a higher gains along with the happy customers.

Here the paper aims in utilizing the big data business analytics in the health care industry to improvise the strategic assets of the health care industry without compromising the patient and the physician satisfaction.

The remaining paper is organized with the section 2. Comprised of the related works, section 3. The proposed work on the big data business analytics with the health care industry to improve its strategic assets. Section 4 . The results holding the improvement in the strategic assets of the industry and user satisfaction and finally the section 5 . Including the conclusion that gives the summary of the work done.

\section{Related works}

Gunasegaram et al [1] the process of big data analytics and the predictive modelling is utilized in the paper to improve the business process in order to achieve a firm performance. Mikalef, et al [2] the paper presents the survey on the big data analytics defining the areas where it could be used. The mechanisms that could help in its implementation and the competitive advantage provided by the big-data analytics. Ji-fan Ren et al [3] the paper is the study based on the big-data values to elaborate the quality and the performance of the business firms. The review also focuses on the completeness, accuracy, of the business using the big data analytics. Côrte-Real [4] the values of the big-data analytics is extracted using the knowledge-based view and DC theories are used to provide with the value for the business. The analysis provided improves the agility of the business performance. Müller et al [5] the paper presents the analytical study of the big data solution in the firm performance and productivity, providing an econometric analysis for big data evolved from the IT business. Krishnan et al [6], the paper explains the need of the big data analytics in the health care to enhance the hospital services showing improvement in the care, coupled with http://irojournals.com/iroismac/ 
Journal of ISMAC (2019)

Vol.01/ No. 02, June 2019, pp: 92-100

ISSN: 2582-1369 (online)

DOI: https://doi.org/10.36548/jismac.2019.2.002

the significant cost savings. Wang et al [7], the paper provides with the comprehensive understanding of the of the potency of the big data analytics in the health care, the paper also provides with the several strides in being successful with the big data analytics in the health care industry. Sindhi, et al [8] the paper presents the review of the health sector of India in the urban as well as the rural areas, and the use of big data in the health care to improve the health care sector in of India. Niu et al [9] the paper gives the valuable information's for the improving the strength of the transmission, the design of the communication system involved in the promoting. The big data provides with the improved decisions for developing of the entire medical system. Bates et al [10] the paper details the prevailing big data methods to improvise the medical care, and the cities adapting to the big data analytics to enhance the heath of the people. Chanchaichujit et al [11] the paper present with the encapsulation of the internet of things and the data analytics health care, data flow in the health and its usage to explain the effective changes it can cause in the health care, the paper also explains the challenges incurred in implementing of the big-data. El Morr et al [12] the need for the analysis of the data and the business intelligence in health care is explained in this paper, the decision making assisted by the data analytics based on the past and the predictive analysis to improve the health care is also explained in it.

\section{Proposed Work}

In the recent days the population of the data flowing through various applications are very much increased compared to the population of the people. The huge amount of data flow contained with the valuable insights that were once considered to be garbage is now gathered and stored to extract the valuable insights to improve the quality of the service and gain the customer relationship. The enormous data flow beyond the storage space of the traditional database leads to the big-data analytics where the big-data helps in accumulating the data, storing them, and analytics help in processing them to extract the valuable insights to improve the smooth and the continuous business operations. The fig. 1 below gives the driving facets of the big-data.

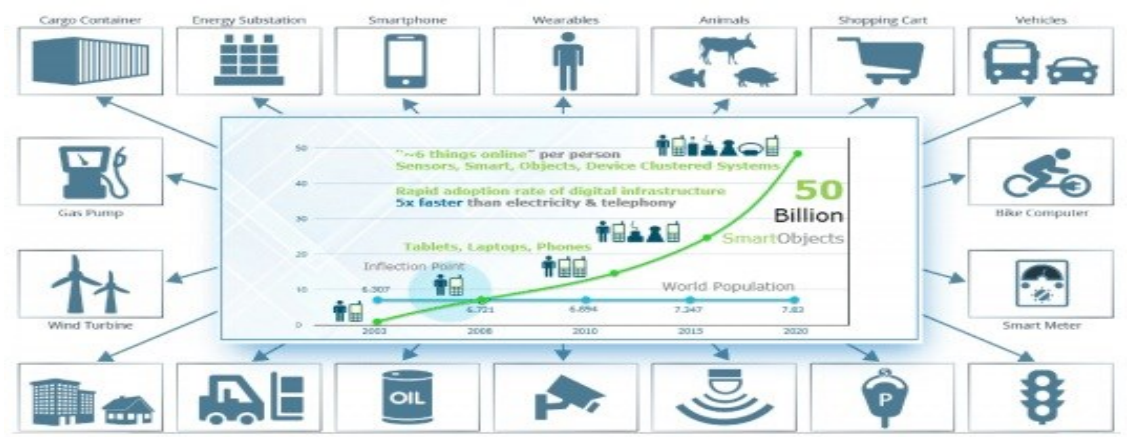

Fig .1 Driving Facets of Big-Data [16].

http://irojournals.com/iroismac/ 
The Fig. 1 shows the few driving facets that are the major sources of the big-data generation, that usually come under the categories such as the enterprise, social, public, transaction and the sensor data's. The paper focuses on the one such facet of big-data the health care industry that provides with the enormous data flow and utilizes the information gathered to identify the leakages in the revenue of the health industry and the inconvenience suffered by the patients and presents with the insights of improving the quality of service of the health care industry to attain the customer satisfaction.

\subsection{Revenue Leakages of the Health care Industry}

The health care industry produces a huge amount of data that are to be monitored in the real time, to improve the quality of the service. Paper considers the revenue leakages in the private hospital, due to its inefficient management and the big-data analytics in improving the strategic assets of the hospital improving the quality of the service it provides and the customer satisfaction. Some of the revenue leakages that bring loss to the health industries are as follows. The failure in the maintenance of the patient records, lack of centralized monitoring on the outpatient and the inpatients, lack maintenance of details of the treatment provided, lack of the facilities and the machineries in diagnosing the disease and the providing the treatment. The fig. 2 shows the revenue leakages in the health-care industry. For e.g. consider in a private hospital without the facilities of the diagnosis (Scan Machine) for the disease. Each time the patient comes for the consulting the doctor he is recommended by the doctor to move to another diagnosis center to know the intense of the disease, this is a revenue loss to the hospital, causing the unwanted drift to 
Journal of ISMAC (2019)

Vol.01/ No. 02, June 2019, pp: 92-100

ISSN: 2582-1369 (online)

DOI: https://doi.org/10.36548/jismac.2019.2.002

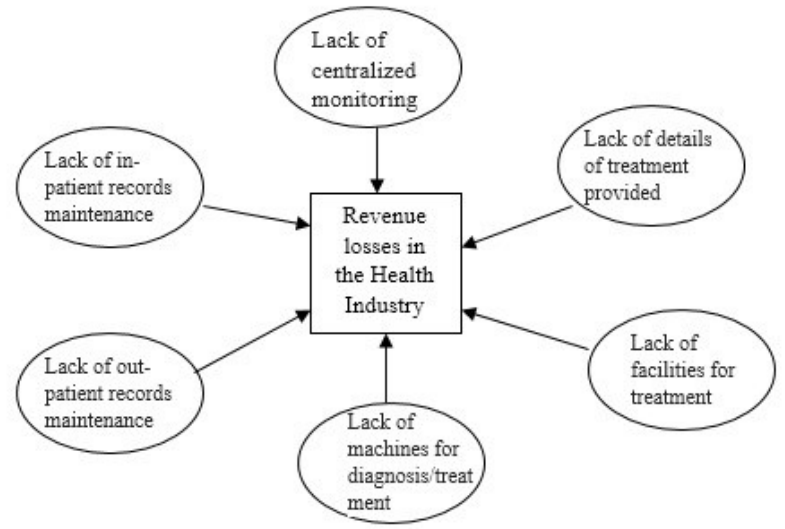

Fig. 2 Revenue loss of the health care Industry

the patients which might even cause the hospital to lose its customers, thus reducing the revenue of the hospital. The example gives one of the revenue losses of the hospital, there are many other reasons also that leads revenue and the customer losses in the hospital.

\subsection{The Big-Data analytics in the improving the Revenue losses}

The big data in order to improve the strategic assets of the hospital involves the following procedures of data identification, accumulation, analysis, visualization and applications of the insights gained to improve the efficiency of the health industry. The flow chart shown below in the fig. 3 explains the process of the big data business analytics improving the strategic assets of the health industry and improving the customer satisfaction.

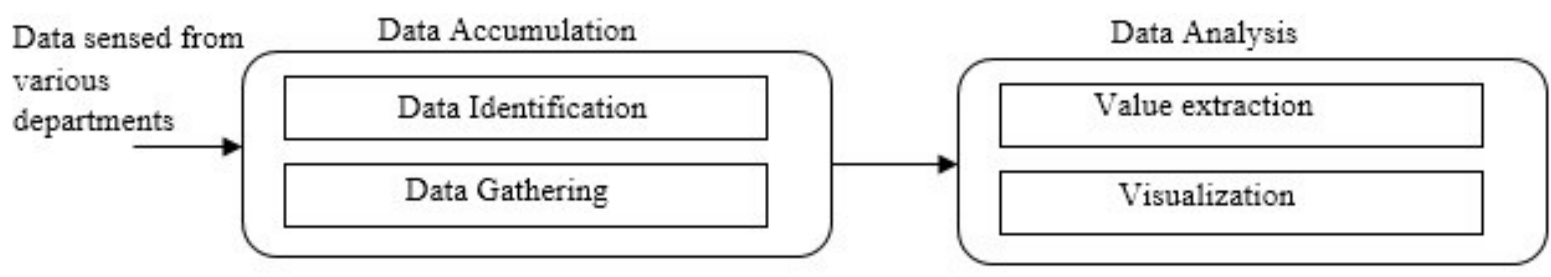

Fig.3 proposed Flow diagram.

\subsubsection{Data Accumulation}

The medical record of the inpatients, the out patients, the doctor prescriptions, recommendations, the patient feedbacks, the infrastructure details of the hospital etc. are gathered further the other areas that cause to the http://irojournals.com/iroismac/ 
Journal of ISMAC (2019)

Vol.01/ No. 02, June 2019, pp: 92-100

DOI: https://doi.org/10.36548/jismac.2019.2.002

ISSN: 2582-1369 (online)

deterioration of the hospital performance leading to the revenue losses are very voluminous are gathered over the local network connection and stored into the Hadoop cloud using the internet for the further processing in the future. The information accumulated and stored into the Hadoop cloud are proceeded with the next process of the analysis that includes the extraction of the valuable information and filtering of the data that are unwanted to gain the insights in developing the strategic assets of the health care industry.

\subsubsection{Data Analysis}

The information stored in the distributed file system of the Hadoop cloud is, analyzed assigning the parallel bat algorithm using the map reduce [15] to filter the data accumulated and sorting out the useful information that are necessary in the improving the services of the health industry and the reducing its revenue losses. For e.g. from the details of the particular in patients gathered based on the treatment provided and the intensity of the disease, gathers the necessary information that are required in the improving the treatment for the disease for the speedy recovery of the patient. The sorted data that are separated from the unwanted data are visualized for the improving the services of the health industry. The necessary insights sought from the data accumulated and analyzed, helps to visualize the counter measures that could improve the standard of the hospital. The fig. 4 below gives the data analysis procedure

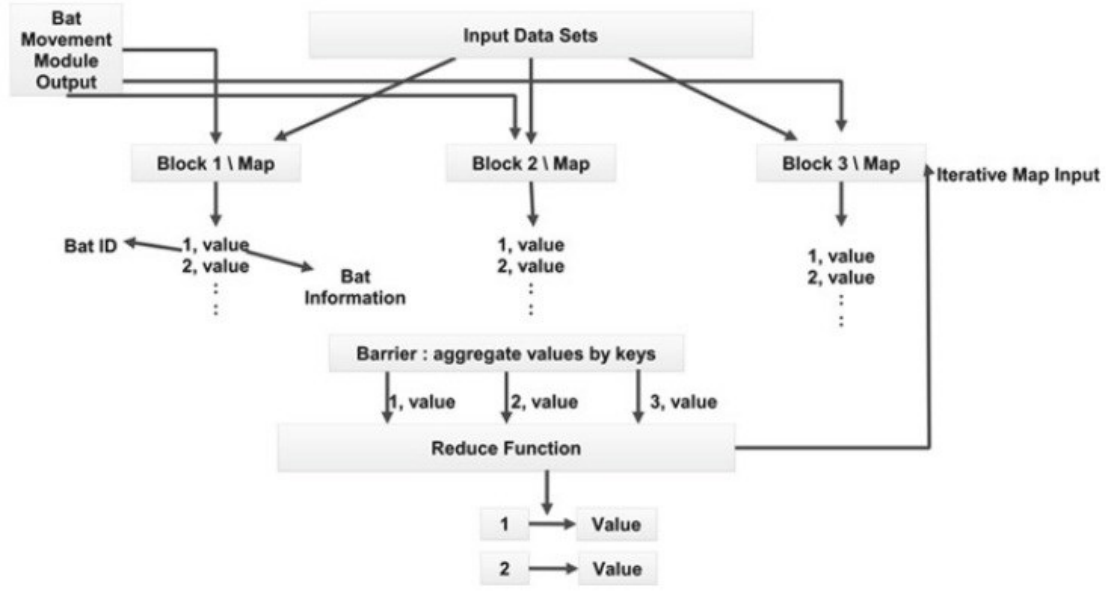

Fig. 4 Data Analysis Procedure [15]

involving the parallel bat algorithm using the map reduce [15] to identifying the values of the information gathered. Thus improving the way of treatment, facilities of treatment (san center, gym, physiotherapy center, pharmacy ), with the centralized monitoring gathering the complete information of the hospital activities and the patients details along with the permanent storage for the each and every details gathered in the Hadoop cloud. The Fig. 5 gives the steps of the parallel BAT algorithm using the map reduce for extracting the important values of the data to improve the strategy assets of the health care industry. 
Journal of ISMAC (2019)

Vol.01/ No. 02, June 2019, pp: 92-100

ISSN: 2582-1369 (online)

DOI: https://doi.org/10.36548/jismac.2019.2.002

\section{Results and Discussion}

The results evaluation of the proposed parallel bat algorithm for the purpose of identifying the values contained in the enormous data set accumulated, shows that the proposed system provides with the better insight on the data accumulated than the other methods based on the k-means and the particle swarm optimization. The insights obtained could help in the improving the hospital standards offering a better quality of service, the fig. 6 shows the percentage of the valuable data attained to enhance the quality of service of the hospital under various departments such as the centralized monitoring of the in patients and the outpatient record, the treatment provided and the infrastructure to be improved.

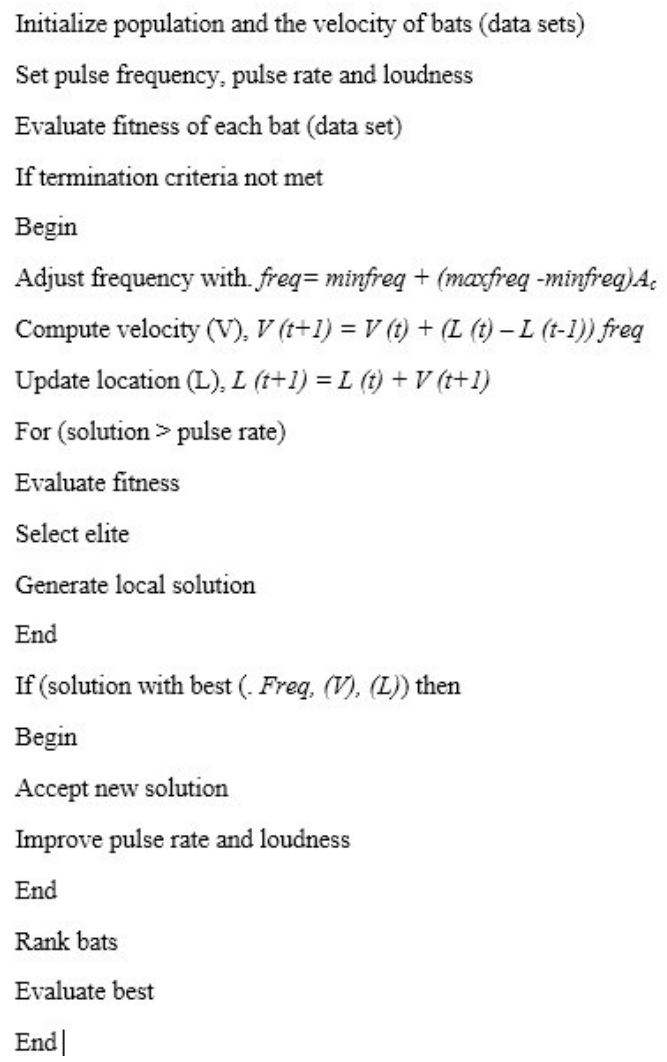

Fig. 5 Parallel BAT algorithm using the map 
Journal of ISMAC (2019)

Vol.01/ No. 02, June 2019, pp: 92-100

DOI: https://doi.org/10.36548/jismac.2019.2.002

ISSN: 2582-1369 (online)

The table. 1 below gives the comparison of the accuracy in predicting the values of the information gathered. The parallel-bat algorithm utilized with the map reduce in deducing the values from the huge amount of data accumulated from the various departments of the hospital to improvises it servicing in turn increasing its revenue, provides with the better accuracy in extracting the values than the other methods of K means [13] and the PSO[14].

\begin{tabular}{|c|c|c|c|c|c|}
\hline $\begin{array}{l}\text { Data Set } \\
\text { Algorithm }\end{array}$ & 100 & 200 & 300 & 400 & 500 \\
\hline K-Means & 35 & 38 & 39 & 42 & 44 \\
\hline PSO & 50 & 53 & 51 & 64 & 65 \\
\hline Proposed & 85 & 88 & 83 & 84 & 90 \\
\hline
\end{tabular}

Table.1 Accuracy Percentage in Predicting Values

Further the method of the parallel bat algorithm for the analysing the value in the accumulated data to identify the method in improving the hospital standards enables to a maximizing the customer satisfaction, by enhancing the ways of treating a disease enabling a faster recovery maintaining the complete information of the treatment provided and regularizing the check-up after delivery. Moreover the information gathered based on the infrastructure of the hospital enables it to have details of the infrastructure available, the things to be updated, service, maintained and newly purchased.

\section{Conclusion}

The paper providing the big-data business analytics for improvising the strategy assets of the health care industries, identifies the causes of the revenue losses and gathers data from the various departments of the hospital to analyze the same using the clustering based on the bat algorithm using the map reduce enables to extract the values contained in the information gathered and stores them permanently in the cloud over the internet. So that they could be retrieved any time they are in need, the values deduced help in the improvising the hospital service meeting the needs of the patients and maintaining the customer relationship in turn improving the strategic assets of the health care industry. The performance assessment of the proposed method based on the parallel-bat algorithm for the deducing the values from the information shows an improvement in percentage of attaining the values from the information and the accuracy of the values gathered compared to the prevailing methods used in the extracting the values from the information.

\section{References}


Journal of ISMAC (2019)

Vol.01/ No. 02, June 2019, pp: 92-100

DOI: https://doi.org/10.36548/jismac.2019.2.002

ISSN: 2582-1369 (online)

[1] Gunasekaran, Angappa, Thanos Papadopoulos, Rameshwar Dubey, Samuel Fosso Wamba, Stephen J. Childe, Benjamin Hazen, and Shahriar Akter. "Big data and predictive analytics for supply chain and organizational performance." Journal of Business Research 70 (2017): 308-317.

[2] Mikalef, Patrick, Ilias O. Pappas, John Krogstie, and Michail Giannakos. "Big data analytics capabilities: a systematic literature review and research agenda." Information Systems and e-Business Management 16, no. 3 (2018): 547-578.

[3] Ji-fan Ren, Steven, Samuel Fosso Wamba, Shahriar Akter, Rameshwar Dubey, and Stephen J. Childe. "Modelling quality dynamics, business value and firm performance in a big data analytics environment." International Journal of Production Research 55, no. 17 (2017): 5011-5026.

[4] Côrte-Real, Nadine, Tiago Oliveira, and Pedro Ruivo. "Assessing business value of Big Data Analytics in European firms." Journal of Business Research 70 (2017): 379-390.

[5] Müller, Oliver, Maria Fay, and Jan vom Brocke. "The effect of big data and analytics on firm performance: An econometric analysis considering industry characteristics." Journal of Management Information Systems 35, no. 2 (2018): 488-509.

[6] Krishnan, Shankar M. "Application of analytics to big data in healthcare." In 2016 32nd Southern Biomedical Engineering Conference (SBEC), pp. 156-157. IEEE, 2016.

[7] Wang, Yichuan, LeeAnn Kung, and Terry Anthony Byrd. "Big data analytics: Understanding its capabilities and potential benefits for healthcare organizations." Technological Forecasting and Social Change 126 (2018): 3-13.

[8] Sindhi, Komal, Dilay Parmar, and Pankaj Gandhi. "A Study on Benefits of Big Data for Healthcare Sector of India." In Data Science and Big Data Analytics, pp. 239-246. Springer, Singapore, 2019.

[9] Niu, Wenjing, Jinyan Huang, Zhao Xing, and Jianbin Chen. "Knowledge Spillovers of Medical Big Data Under Hierarchical Medical System and Patients' Medical Treatment Decisions." IEEE Access 7 (2019): 55770-55779.

[10] Bates, David W., Axel Heitmueller, Meetali Kakad, and Suchi Saria. "Why policymakers should care about "big data" in healthcare." Health Policy and Technology 7, no. 2 (2018): 211-216.

[11]Chanchaichujit, Janya, Albert Tan, Fanwen Meng, and Sarayoot Eaimkhong. "Internet of Things (IoT) and Big Data Analytics in Healthcare." In Healthcare 4.0, pp. 17-36. Palgrave Pivot, Singapore, 2019.

[12]El Morr, Christo, and Hossam Ali-Hassan. "Healthcare, Data Analytics, and Business Intelligence." In Analytics in Healthcare, pp. 1-13. Springer, Cham, 2019.

[13] Cui, Xiaoli, Pingfei Zhu, Xin Yang, Keqiu Li, and Changqing Ji. "Optimized big data K-means clustering using MapReduce." The Journal of Supercomputing 70, no. 3 (2014): 1249-1259.

[14]A. W. McNabb, C. K. Monson, and K. D. Seppi, "Parallel pso using mapreduce," in Evolutionary Computation, 2007. CEC 2007. IEEE Congress on, 2007.

[15]Ashish, Tripathi, Sharma Kapil, and Bala Manju. "Parallel bat algorithm-based clustering using mapreduce." In Networking Communication and Data Knowledge Engineering, pp. 73-82. Springer, Singapore, 2018.

http://irojournals.com/iroismac/ 
Journal of ISMAC (2019)

Vol.01/ No. 02, June 2019, pp: 92-100

DOI: https://doi.org/10.36548/jismac.2019.2.002

ISSN: 2582-1369 (online)

[16]https://www.edureka.co/blog/big-data-tutorial

http://irojournals.com/iroismac/ 